\title{
Endocrinological responses during suckling in Hatano high- and low-avoidance rats
}

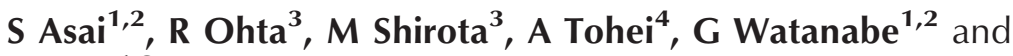 \\ K Taya ${ }^{1,2}$ \\ ${ }^{1}$ Department of Basic Science, United Graduate School of Veterinary Sciences, Gifu University, Gifu, Gifu 501-1193, Japan \\ ${ }^{2}$ Laboratory of Veterinary Physiology, Tokyo University of Agriculture and Technology, Fuchu, Tokyo 183-8509, Japan \\ ${ }^{3}$ Safety Testing Laboratory, Research Administration, Hatano Research Institute, Food and Drug Safety Center, Hatano, Kanagawa 257-8523, Japan \\ ${ }^{4}$ Laboratory Animal Research Center, Dokkyo University School of Medicine, Mibu, Tochigi 321-0293, Japan \\ (Requests for offprints should be addressed to K Taya, Laboratory of Veterinary Physiology, Department of Veterinary Medicine, Faculty of Agriculture, Tokyo \\ University of Agriculture and Technology, 3-5-8 Saiwai-cho, Fuchu, Tokyo 183-8509, Japan; Email: taya@cc.tuat.ac.jp)
}

\begin{abstract}
Hatano high-avoidance (HAA) and low-avoidance (LAA) animals were originally selected from Sprague-Dawley rats for good and poor active avoidance learning in a shuttle box. We studied the endocrinological profile in lactating rats to determine the effect of suckling during midlactation in HAA and LAA rats. The pups were separated from their mother rats $6 \mathrm{~h}$ before the onset of suckling and blood samples were drawn from unanaesthetized mother rats via a jugular cannula at 0,5 and $15 \mathrm{~min}$ after the suckling stimulus and then 15, 45 and 105 min after pups were removed.

Plasma concentrations of oxytocin in HAA rats were significantly higher than in LAA rats during the suckling period. Plasma concentrations of prolactin and ACTH in HAA rats were significantly higher than in LAA rats during the suckling period, and at $15 \mathrm{~min}$ and $45 \mathrm{~min}$ after the pups were removed. However, there were no strain differences in circulating corticosterone between the two lines, indicating that the response of the hypothalamopituitary axis to the suckling stimulus was greater in HAA
\end{abstract}

rats than in LAA rats, whereas the ACTH-induced adrenal response of corticosterone release was higher in LAA rats than in HAA rats. Since dopamine from the median eminence inhibits prolactin secretion from the lactotrophs of the anterior pituitary, and tuberoinfundibular dopaminergic neurones are partially regulated by the level of circulating prolactin, we evaluated the activity of tyrosine hydroxylase $(\mathrm{TH})$, the rate-limiting enzyme in dopamine biosynthesis. TH, measured by the accumulation of 3,4dihydroxyphenylalanine, was significantly higher in HAA rats than in LAA rats before the suckling stimulus. After the suckling stimulus, TH activity in HAA rats was significantly lower than before suckling, whereas $\mathrm{TH}$ activity in LAA rats was not changed.

These findings clearly demonstrated that apparent differences between the two Hatano lines exist in endocrinological profiles during suckling. These strain differences probably originate from neurotransmitter changes, such as dopamine.

Journal of Endocrinology (2004) 182, 267-272

\section{Introduction}

Pups obtain necessary nourishment and cause a variety of hormonal changes in the mother by suckling (Voogt 1978). The suckling stimulus increases the plasma concentrations of prolactin (Taya \& Greenwald 1982, Grosvenor et al. 1986, Taya \& Sasamoto 1989, 1990), adrenocorticotrophic hormone (ACTH), corticosterone (Voogt et al. 1969) and oxytocin (Higuchi et al. 1985, 1986, Grosvenor et al. 1986), and decreases luteinizing hormone and follicle-stimulating hormone (Taya \& Greenwald 1982, Taya \& Sasamoto 1989, 1990).

The Hatano rat lines have been genetically selected and bred from Sprague-Dawley rats to estimate the pharmacological and toxicological effects of chemicals using the performance shuttle-box test. This learning test assesses the acquisition of avoidance ability by the rats escaping to the safe chamber after a conditioned stimulus of sound and light. When animals fail to avoid the stimulus they receive an electric shock. The Hatano rat lines were selected to resolve the high variability in the data using this test. High-avoidance animals (HAA) quickly acquire the active avoidance response, whereas low-avoidance rats (LAA) fail to acquire this response (Ohta et al. 1995). Besides the divergence in active avoidance behaviour, we have shown various other phenotypic differences. Adrenal weights are heavier in HAA rats than in LAA rats, and plasma concentrations of ACTH are higher in HAA rats 
than in LAA rats(Ohta et al. 1999). HAA rats and LAA rats show differences in follicular development, luteal function and pattern of hormonal secretion during the oestrous cycle (Asai et al. 2002). The body weights of the HAA pups are heavier than the LAA pups during the latter half of lactation. HAA dams retrieve their pups faster and eject more milk than LAA dams (Ohta et al. 2002).

However, differences in the endocrinological profiles during suckling between the two Hatano lines have not been studied. To test the hypothesis that strain differences exist in the profiles of the hypothalamo-pituitary-adrenal axis and milk secretion after a suckling stimulus in Hatano rat lines, we examined the secretory pattern of plasma hormones after the suckling stimulus and the activity of the rate-limiting enzyme for dopamine synthesis, tyrosine hydroxylase $(\mathrm{TH})$, in the stalk-median eminence (SME) in order to investigate the regulation of prolactin secretion.

\section{Materials and Methods}

\section{Animals}

Nulliparous female HAA rats $(n=20)$ and LAA rats $(n=20)$ at 70-100 days of age were used. Animals were maintained under a $12 \mathrm{~h}$ light: $12 \mathrm{~h}$ darkness cycle (light period from 0700 to $1900 \mathrm{~h}$ ), at a temperature of $21-25^{\circ} \mathrm{C}$ and relative humidity of 50-65\%. Food (CE-2; Clea Japan, Inc., Tokyo, Japan) and water were available ad libitum. Mating was allowed to occur within each strain. All pregnant rats were housed individually in plastic cages $(35 \times 40 \times$ $18 \mathrm{~cm}$ ) with wood chip bedding and allowed to deliver spontaneously. The litter size was adjusted to eight pups per dam on lactation day 1 (day of parturition=day 0 of lactation). All experimental procedures involving animals were carried out in accordance with The Guide for the Care and Use of Laboratory Animals prepared by Tokyo University of Agriculture and Technology, and approved by the Animal Care Use Committee at the Hatano Research Institute of Food and Drug Safety Center.

Plasma hormonal responses after the suckling stimulus in lactating rats

One day before each experiment (lactation day 12-14), a silicon cannula (Kaneka Medix Co., Osaka, Japan) was inserted into the atrium via the external jugular vein in each rat under ether anaesthesia, to be used to withdraw blood samples. The cannula was kept patent with heparinized saline whenever blood was not being removed.

On lactation day 13-15, the pups were separated from their mothers at $6 \mathrm{~h}$ before the onset of suckling. Blood samples were drawn from a jugular cannula at 0,5 and 15 min after suckling began, followed by sampling at 15 ,
45 and 105 min after the pups were removed. Blood was collected in heparinized tubes containing aprotinin and centrifuged immediately, and plasma was separated and stored at $-60{ }^{\circ} \mathrm{C}$ until assayed for $\mathrm{ACTH}$, corticosterone, prolactin and oxytocin.

\section{$R I A$}

Plasma concentrations of ACTH (Kanesaka et al. 1992) and corticosterone (Tomabechi et al. 1994) in plasma were measured by double-antibody RIAs using ${ }^{125}$ I-labelled radioligands as described previously. Synthetic rat ACTH 1-39 (Sigma Chemical Co., St Louis, MO, USA) was used as the reference standard. The intra- and interassay coefficients of variation were $11.3 \%$ and $11.9 \%$ for ACTH and $9.5 \%$ and $16 \cdot 4 \%$ for corticosterone respectively.

Plasma concentrations of oxytocin were also measured by double-antibody RIAs using ${ }^{125}$ I-labelled radioligands. Synthetic oxytocin (Sigma Chemical Co.) was used as the reference standard. Anti-oxytocin serum (rabbit) was purchased from Chemicon International, Inc. (Temecula, CA, USA), and the radioligand oxytocin (NEX 187) was purchased from New England Life Science Products, Inc. (Boston, MA, USA). Plasma samples were purified and separated from plasma proteins using Sep-Pak Plus $\mathrm{C}_{18}$ cartridges (Waters Co., Milford, MA, USA) as described previously (Stock \& Uvnas-Moberg 1988). The intra- and interassay coefficients of variation for oxytocin were $4 \cdot 4 \%$ and $6 \cdot 8 \%$ respectively.

Plasma concentrations of prolactin were measured using NIDDK kits (NIH, Bethesda, MD, USA) for rat prolactin. Hormone for iodination was rat prolactin-I-5. The antiserum used was anti-prolactin-S-9. Results are expressed in terms of NIDDK rat prolactin-RP-2. The intra- and interassay coefficients of variation for prolactin were $3 \cdot 4 \%$ and $5 \cdot 2 \%$ respectively.

\section{TH activity before and after the suckling stimulus in lactating} rats

Mother rats were injected with $m$-hydroxybenzylhydrazine dihydrochloride (NSD 1050; $50 \mathrm{mg} / \mathrm{kg}$ body weight i.p.; Sigma), an L-aromatic amino acid decarboxylase inhibitor, at 30 or $15 \mathrm{~min}$ before the onset of suckling. Rats were killed by decapitation $30 \mathrm{~min}$ after administration of NSD 1050. The SME was dissected with a pair of fine scissors under a stereoscopic microscope, homogenized in $150 \mu \mathrm{l} 0 \cdot 1 \mathrm{M}$ perchloric acid, and centrifuged at $10000 \mathrm{~g}$ for $10 \mathrm{~min}$ (Demarest 1980). The content of 3,4-dihydroxyphenylalanine (DOPA) in the supernatant was determined by HPLC with electrochemical detection. The pellet was solubilized in $0.5 \mathrm{M}$ PBS and analysed for protein content by the method of Bradford (Bradford 1976).

\section{Milk ejection and pup weight}

All pups were removed from their dams on the morning of the experiment (lactation day 13-15). After $6 \mathrm{~h}$ of 


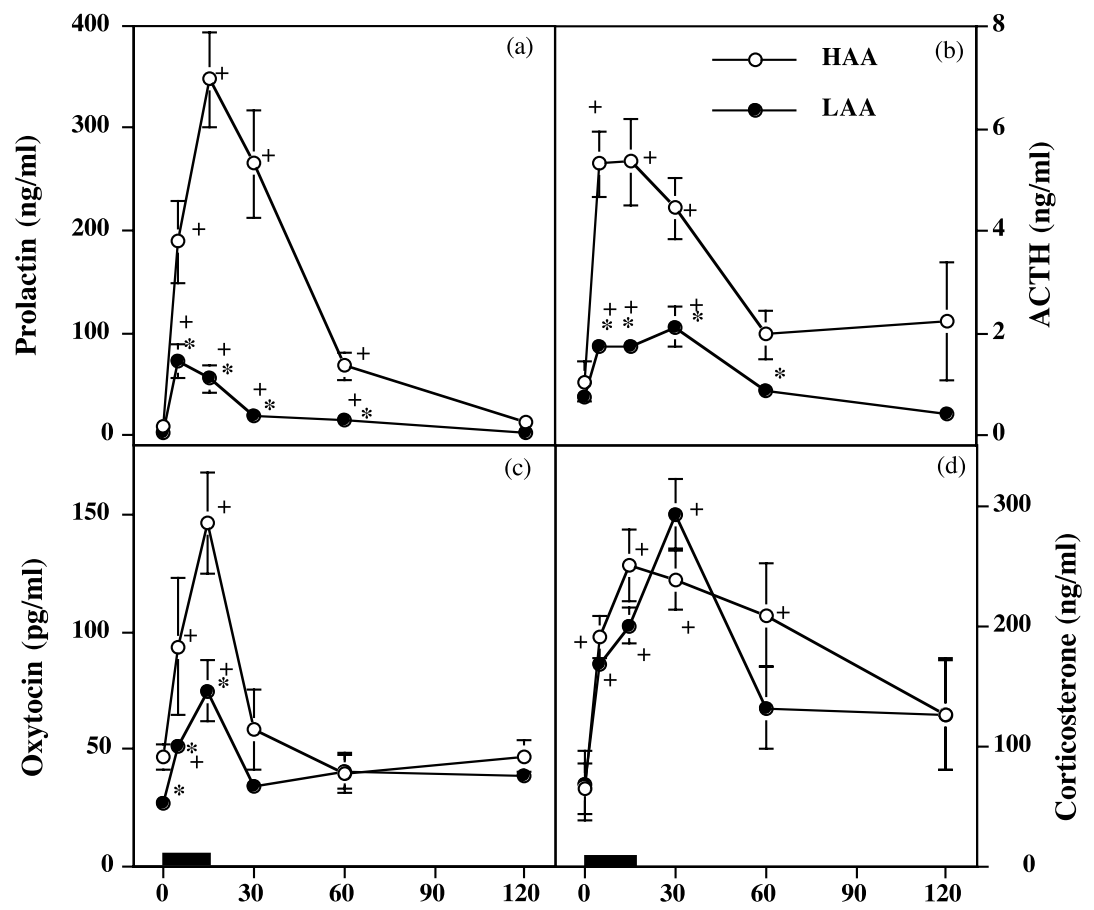

Figure 1 Effects of the suckling stimulus (solid bars) on plasma concentrations of (a) prolactin, (b) ACTH, (c) oxytocin and (d) corticosterone in HAA and LAA rats. Data are expressed as the means \pm S.E.M. of six rats. ${ }^{*} P<0.05$ versus HAA rats (Student's $t$-test or Mann-Whitney $U$ tests). ${ }^{+} P<0 \cdot 05$ versus presuckling values (two-way ANOVA followed by Tukey-Kramer tests).

isolation, urine was manually expressed from the bladders of the pups. Pups were weighed and returned to their dams for $15 \mathrm{~min}$ of suckling and then reweighed to determine the amount of milk yield they had obtained from the dam.

\section{Statistical analyses}

All values are expressed as means \pm S.E.M. Significant differences were analysed between HAA rats and LAA rats by Student's $t$-test when uniformity of variance was confirmed by F-test. When the variance was not uniform, Mann-Whitney U test was used. Plasma concentrations of prolactin, ACTH, corticosterone and oxytocin, and concentrations of DOPA were analysed using two-way ANOVA followed by Tukey-Kramer test. $P<0.05$ was considered statistically significant.

\section{Results}

Circulating hormone levels after the suckling stimulus in lactating rats (Fig. 1)

The suckling stimulus induced an increase in plasma concentrations of prolactin, ACTH, oxytocin and corticosterone in both strains. The plasma concentrations of prolactin and ACTH in HAA rats were significantly higher than in LAA rats during the suckling period and 15 and $45 \mathrm{~min}$ after the pups were removed (Fig. 1a and b). Plasma oxytocin levels before the suckling stimulus in HAA rats were significantly higher than in LAA rats. Concentrations of plasma oxytocin in HAA rats were significantly higher than in LAA rats during the suckling period (Fig. 1c). There was no strain differences in the concentrations of corticosterone between the two lines during the period studied (Fig. 1d).

TH activity in the SME before and after the suckling stimulus (Fig. 2)

The HAA and LAA rats received the i.p. injection of NSD 1050 either $30 \mathrm{~min}$ before the return of the pups or $15 \mathrm{~min}$ before the onset of suckling followed by suckling for $15 \mathrm{~min}$. In all cases, rats were killed $30 \mathrm{~min}$ after NSD 1050 was injected. TH activity was measured by the accumulation of DOPA at $30 \mathrm{~min}$ after an injection of NSD 1050. TH activities before the suckling stimulus in HAA rats were significantly higher than in LAA rats. After the suckling stimulus, TH activity in HAA rats was significantly lower than before suckling, whereas TH activity in LAA rats was not changed compared with that before suckling. 


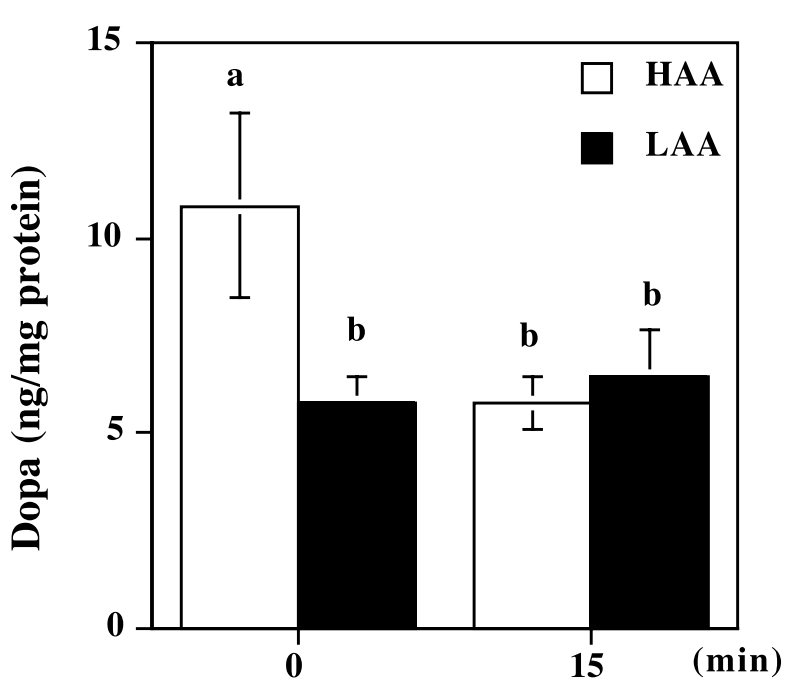

Time after the onset of suckling

Figure 2 DOPA accumulation at $30 \mathrm{~min}$ after an injection of NSD 1050 in lactating HAA and LAA rats. Rats received the injection of NSD 105015 min before the onset of suckling and then suckling was allowed for $15 \mathrm{~min}$. Data are expressed as the means \pm S.E.M. of seven rats. Bars with different letters are significantly different from one another at $P<0.05$ as determined by two-way ANOVA followed by the Tukey-Kramer test.

Milk ejection and body weight (Fig. 3)

Milk yield was significantly lower for LAA rats than for HAA rats. Body weights of LAA pups were lower than HAA rats after the experiment.

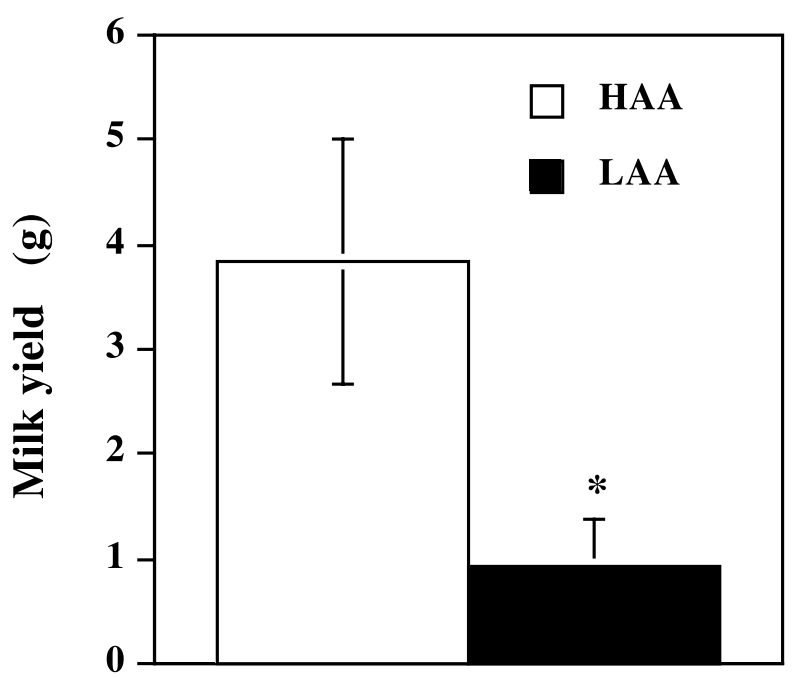

Figure 3 Milk yield in HAA and LAA dams determined by gain in pup weight during a 15 -min period of suckling following a $6-\mathrm{h}$ separation ${ }^{*} P<0 \cdot 05$ versus HAA rats (Student's $t$-test).
Correlations between ACTH and corticosterone (Fig. 4)

There was a positive correlation between plasma concentrations of ACTH and corticosterone in HAA rats $(n=6$, $\left.\mathrm{r}^{2}=0 \cdot 63, P<0 \cdot 05\right)$ and LAA rats $\left(n=6, \mathrm{r}^{2}=0 \cdot 38, P<0 \cdot 05\right)$. The data showed that there was a clear difference between the two strains in the stress response to the suckling stimulus of the hypothalamo-pituitary and adrenal glands in the secretion of ACTH and corticosterone respectively. The hypothalamo-pituitary response of ACTH secretion to the suckling stimulus in HAA rats was higher than in LAA rats, whereas the adrenal response of corticosterone secretion was higher in LAA rats than in HAA rats.

\section{Discussion}

The present study clearly demonstrated that there are differences between HAA rats and LAA rats in the pattern of hormonal secretion during suckling. The concentrations of plasma prolactin, ACTH and oxytocin in LAA rats were significantly lower than in HAA rats during the suckling period. The weights of LAA pups were lower than those of HAA rats. Milk yield was significantly lower in LAA rats than in HAA rats. Our previous report showed LAA pups with reduced weight gain compared with HAA pups (Ohta et al. 1998), LAA dams with a lowered prolactin secretion 15 min after suckling compared with HAA rats, and LAA dams with a lowered milk yield during a 15-min period of suckling compared with HAA dams (Ohta et al. 2002). We also reported that there were no strain differences in the levels of milk ejection following oxytocin injection between HAA and LAA rats (Ohta et al. 2002). Furthermore, we investigated the weight of the pups in a previous cross-fostering study (Ohta et al. 1998) where half the litters from HAA dams were fostered onto LAA dams, and half the litters from LAA dams were fostered onto HAA rats. Body weight was lower for HAA offspring fostered by LAA dams than for pups fostered by HAA dams, whereas it was greater for LAA offspring fostered by HAA dams than pups fostered by LAA dams (Ohta et al. 1998). The present study in conjunction with two previous papers (Ohta et al. 1998, 2002) findings suggested that there are no strain differences in milk synthesis and strength of suckling by pups but the differences indicated that LAA rats are more insensitive to the suckling stimulus than HAA rats.

$\mathrm{TH}$ activity was higher in HAA than in LAA rats before the onset of suckling. TH activity decreased at $15 \mathrm{~min}$ after the onset of suckling in HAA rats, whereas it did not change in LAA rats in response to suckling. Prolactin acts on the epithelial cells of the mammary gland to stimulate milk synthesis. Complex neuronal pathways involved in translating the suckling stimulus to increased prolactin secretion exist. It is well known that dopamine is a physiologically important prolactin-inhibitory factor and is secreted into hypothalamic portal blood to inhibit 


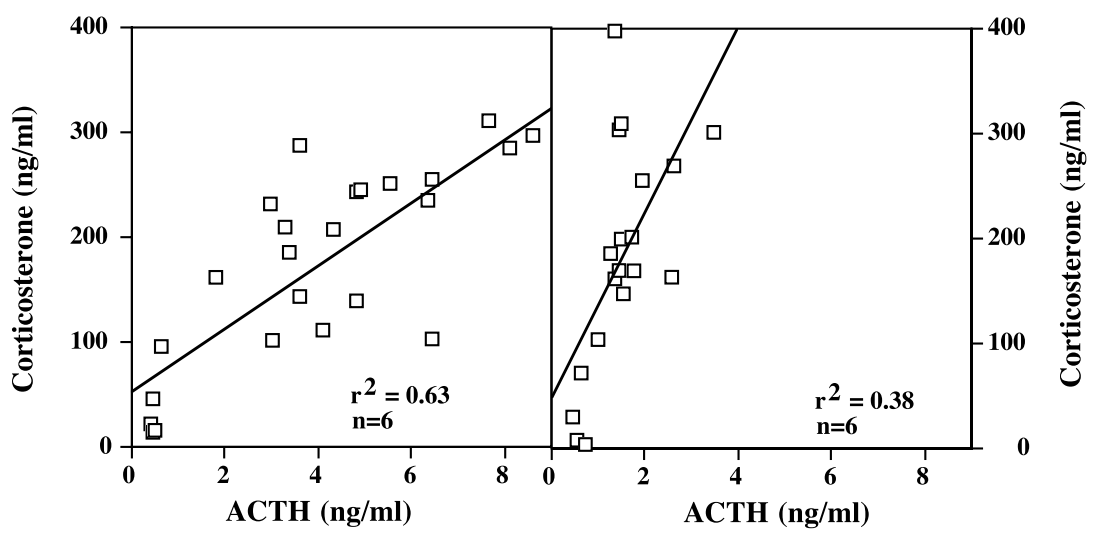

Figure 4 Correlation between plasma concentrations of ACTH and corticosterone in HAA rats (left-hand graph) and LAA rats (right-hand graph) during suckling and at 15 min after suckling. The number of animals $(n)$ and correlation coefficients $\left(r^{2}\right)$ are as indicated.

prolactin release from lactotrophs tonically (MacLeod \& Login 1976, Matsuzaki et al. 1997). In early lactation, the prolactin-induced increase in $\mathrm{TH}$ activity leads to negative feedback, but this effect is lost by mid-lactation. The injection of MMQ cells (which are anterior pituitary tumour cells known to secrete large amounts of prolactin in the rat) inhibited plasma prolactin levels in dams on day 6 of lactation, but not on day 13 (Arbogast \& Vogt 1996). Treatment with an s.c. injection of prolactin increased TH activity in the SME on day 6, but not on day 13 (Voogt et al. 2001). Twenty-four hours after pup removal, the tuberoinfundibular dopamine neuronal activity and $\mathrm{TH}$ mRNA expression increased. Following removal of the suckling stimulus, prolactin decreased via changes in the activity of tuberoinfundibular dopamine neurones (Dermarest \& Moore 1980, Arbogast \& Voogt 1996). Removal of tonic dopamine inhibition is not sufficient to account for the high levels of prolactin attained during lactation, and additional releasing factors are probably involved. Thyrotrophin-releasing hormone, vasoactive intestinal peptide and oxytocin are some of the mediators of the prolactin response induced by suckling (de Greef et al. 1981, 1987, Jaworski et al. 1997, Matsuzaki et al. 1997, Watanobe et al. 2000). In this study, no clear differences between the strains were found in concentrations of prolactin before the suckling stimulus, but $\mathrm{TH}$ activity in HAA rats was significantly higher than in LAA rats. Suckling generally increases dopamine activity after pup removal. However, dopamine activity in LAA rats did not increase after pup removal. These strain differences in prolactin response may be due to regulation of the prolactin-inhibiting factor, dopamine, in addition to the prolactin-releasing factor during lactation. Futher studies are necessary to clarify the differences in the mechanisms.

In conclusion, the results of the present study indicated clearly that HAA and LAA rats exhibit marked differences in the response of prolactin, oxytocin and ACTH secretion during suckling.

\section{Acknowledgements}

We express our gratitude to Dr Reinhold J Hutz (Department of Biological Science, University of Wisconsin-Milwaukee, Milwaukee, WI, USA) for reading the original manuscript and for valuable suggestions. We are grateful to the rat Pituitary Hormone Distribution Program and Dr A F Parlow, NIDDK for providing the RIA materials for rat prolactin.

\section{Funding}

This work was supported by a grant-in-aid for Scientific Research (The 21st Century Center of Excellence Program, E-1) and a Grant-in-Aid for Scientific Research (No. 13660296) from the Ministry of Education, Culture, Sports, Science and Technology of Japan.

\section{References}

Arbogast LA \& Voogt JL 1996 The responsiveness of tuberoinfundibular dopaminergic neurons to prolactin feedback is diminished between early lactation and midlactation in the rat. Endocrinology 137 47-54.

Asai S, Ohta R, Shirota M, Sato M, Watanabe G \& Taya K 2002 Reproductive endocrinology in Hatano high- and low-avoidance rats during the estrous cycle. Endocrine 18 161-166.

Bradford MM 1976 A rapid and sensitive method for the quantitation of microgram quantities of protein utilizing the principle of protein-dye binding. Analytical Biochemistry 72 $248-254$

Demarest KT \& Moore KE 1980 Accumulation of L-dopa in the median eminence: an index of tuberoinfundibular dopaminergic nerve activity. Endocrinology 106 463-468.

de Greef WJ, Plotsky PM \& Neill JD 1981 Dopamine levels in hypophysial stalk plasma and prolactin levels in peripheral plasma of the lactating rat: effects of a simulated suckling stimulus. Neuroendocrinology 32 229-233.

de Greef WJ, Voogt JL, Visser TJ, Lamberts SW \& van der Schoot P 1987 Control of prolactin release induced by suckling. Endocrinology $121316-322$. 
Grosvenor CE, Shyr SW, Goodman GT \& Mena F 1986 Comparison of plasma profiles of oxytocin and prolactin following suckling in the rat. Neuroendocrinology 43 679-685.

Higuchi T, Honda K, Fukuoka T, Negoro H \& Wakabayashi K 1985 Release of oxytocin during suckling and parturition in the rat. Journal of Endocrinology 105 339-346.

Higuchi T, Tadokoro Y, Honda K \& Negoro H 1986 Detailed analysis of blood oxytocin levels during suckling and parturition in the rat. Journal of Endocrinology 110 251-256.

Jaworski RP, Callahan P \& Janik J 1997 Immunoneutralization of beta-endorphin blocks prolactin release during suckling without affecting tuberoinfundibular dopaminergic neural activity. Life Science 61 1301-1311.

Kanesaka T, Taya K \& Sasamoto S 1992 Radioimmunoassay of corticosterone using ${ }^{125} \mathrm{I}$-labeled radioligand. Journal of Reproduction and Development 38 85-89.

MacLeod RM \& Login I 1976 Control of prolactin secretion by the hypothalamic catecholamines. Advances in Sex Hormone Research 2 211-231.

Matsuzaki T, Irahara M \& Aono T 1997 Physiology and action of prolactin. Nippon Rinsho $\mathbf{5 5}$ 2871-2875.

Ohta R, Matumoto A, Hashimoto Y, Nagao T \& Mizutani M 1995 Behavioral characteristics of rats selectively bred for high and low avoidance shuttlebox response. Congenital Anomalies $\mathbf{3 5}$ 223-229.

Ohta R, Matumoto A, Nagao T \& Mizutani M 1998 Comparative study of behavioral development between high and low shuttlebox avoidance rats. Physiology and Behavior 63 545-551.

Ohta R, Shirota M, Adachi T, Tohei A \& Taya K 1999 Plasma ACTH levels during early, two-way avoidance acquisition in highand low-avoidance rats (Hatano strains). Behavior Genetics 29 137-144.

Ohta R, Shirota M, Tohei A \& Taya K 2002 Maternal behavior, milk ejection, and plasma hormones in Hatano high- and low-avoidance rats. Hormones and Behavior 42 116-125.
Sato M, Ohta R, Kojima K \& Shirota M 2002 Differences in sperm motion between high- and low-shuttlebox avoidance rats (Hatano strains). Journal of Andrology 23 250-258.

Stock S \& Uvnas-Moberg K 1988 Increased plasma levels of oxytocin in response to afferent electrical stimulation of the sciatic and vagal nerves and in response to touch and pinch in anaesthetized rats. Acta Physiologica Scandinavica 132 29-34.

Taya K \& Greenwald GS 1982 Mechanisms of suppression of ovarian follicular development during lactation in the rat. Biology of Reproduction 27 1090-1101.

Taya K \& Sasamoto S 1989 Inhibitory effects of corticotrophinreleasing factor and $\beta$ - endorphin on LH and FSH secretion in the lactating rat. Journal of Endocrinology 120 509-515.

Taya K \& Sasamoto S 1990 Involvement of the adrenal gland in the suckling-induced decrease in LH and FSH secretion and the concomitant increase in prolactin secretion in the rat. Journal of Endocrinology 125 279-285.

Tomabechi T, Taya K, Akai M \& Sasamoto S 1994 A radioimmunoassay for adrenocorticotropic hormone $(\mathrm{ACTH})$ in unextracted plasma of various animals. Journal of Reproduction and Development 40 99-104.

Voogt JL 1978 Control of hormone release during lactation. Clinical Obstetrics and Gynecology 5 435-455.

Voogt JL, Sar M \& Meites J 1969 Influence of cycling, pregnancy, labor, and suckling on corticosterone-ACTH levels. American Journal of Physiology 216 655-658.

Voogt JL, Lee Y, Yang S \& Arbogast L 2001 Regulation of prolactin secretion during pregnancy and lactation. Progress in Brain Research 133 173-185.

Watanobe H, Schioth HB, Wikberg JE \& Suda T 2000 Evaluation of the role for prolactin-releasing peptide in prolactin secretion induced by ether stress and suckling in the rat: comparison with vasoactive intestinal peptide. Brain Research 865 91-96.

Received 30 April 2004

Accepted 11 May 2004 